\title{
Aspergilloma in the frontal sinus expanding into the orbit
}

\author{
H Swoboda, R Ullrich
}

\begin{abstract}
A case of primary frontal sinus aspergilloma in a 79 year old non-immunocompromised woman, who presented with a right sided pyocele expanding into the orbit, is presented. The low susceptibility of the frontal sinus is probably related to the brachycephalic shape of the human skull which locates this sinus far anterosuperiorly to the nasal cavity. In human frontal sinus aspergillosis nasal symptoms are absent; the clinical manifestation of this rare disease is initiated by complications, especially orbital or intracranial invasion.
\end{abstract}

Sinus opacity may raise early suspicion. Treatment consists of surgical debridement and re-aeration. Diagnosis is established by histological analysis of intraluminal contents.

The fungal genus Aspergillus from the order of Ascomycetes comprises a multitude of species and varieties of ubiquitous and omnivorous saprophytic fungi which decompose necrobiotic material, mainly of vegetal origin. ${ }^{12} \mathrm{~A}$ few, mostly thermophilic species occasionally become pathogenic to man and various animal species. Inhalation of the airborne conidia may lead to fungal colonisation of preformed cavities of the respiratory tract. Local tissue damage or pathological secretion as well as general immune deficiency facilitate the progression into distinct, though interrelated, forms of mycoses: non-invasive, invasive, and fulminant aspergillosis. Other pathogenic effects related to aspergillus are allergy and production of carcinogenic aflatoxins. ${ }^{1-3}$

In 1931 Just reported a case of rhinogenic brain aspergillosis with spread into the ethmoid and frontal sinus. ${ }^{3}$ Expansion from ethmoid affection into a frontal sinus was mentioned in three other instances. ${ }^{4-6}$ Gupta (1973) was the first to report primary frontal sinus aspergillosis, ${ }^{7}$ followed by three others. $^{8-10}$

Otorhinolaryngology,

University of Vienna,

Austria

H Swoboda

Department of Pathology, University of Vienna, Austria R Ullrich

Correspondence to: Dr H Swoboda, 1 HNO-Klinik der Universitä Wien, Lazarettgasse 14, A-1090 Wien, Austria

Accepted for publication 30 October 1991

\section{Case report}

A 79 year old woman presented with right sided proptosis with sudden onset one week previously and epiphora for two weeks. Nasal obstruction or discharge or epistaxis had never occurred. General condition, body temperature, chest radiographs and routine laboratory exams were normal except for an increased erythrocyte sedimentation rate $(52 / 70)$. Deep in the upper eyelid a smooth but very firm painless resistance was palpable, slightly displacing the ocular bulb. The nasal cavities were inconspicuous.

Plain radiographs and conventional tomograms showed a lowered, flattened and thinned orbital roof on the right, inhomogenous opacity of both frontal sinuses, and hyperostosis frontalis interna. All other sinuses showed normal radiotranslucency. An ultrasound scan showed pathological ultrasonographic transmittance of the lowered orbital roof. Trepanation of the right frontal sinus showed a pyocele containing a yellowish bean-sized concrement. The lateral two thirds of the floor of the sinus were defective. After resection of heavily inflammed mucosa and drainage into the nasal cavity the site was closed.

Histological examination of the mucosa showed only non-specific inflammation with shallow ulcerations. The concrement showed lamellar stratification and consisted of inflammatory detritus densely infiltrated by fungal mycelia. Hyphae were septated, dichotomously ramified, and terminated into vesicular fruit heads (figure). This morphology was consistent with an Aspergillus mycetoma. Culture failed, therefore the fungus could not be specified further. The postoperative course was uneventful. Clinical and serological signs of inflammation regressed slowly; Aspergillus haemagglutination tests were negative. Ophthalmological controls confirmed the patency of lacrimal draining pathways. No sign of recurrence was seen in computed tomogram taken three months postoperatively.

\section{Discussion}

Non-invasive aspergillosis usually affects immunologically competent individuals, and depends essentially on local host conditions. Prolonged exposure to contaminated vegetable detritus adds a secondary risk factor as suggested by a high incidence in southern countries with a warm and humid climate. ${ }^{25-8}$

The paranasal sinuses offer the fungus good conditions for subsistance, and chronic infection may lead to the development of mycetoma. Chronicity of sinusitis results from inflammatory mucosal swelling around the ostia hampering ventilation and drainage. Ensuing dyschylia and mucociliary stasis provide the fungus with nutrients, and anaerobic conditions with environmental acidity foster hyphal growth. The susceptibility of the different sinuses to chronic infection and thus to aspergillosis varies greatly: either disease predom- 

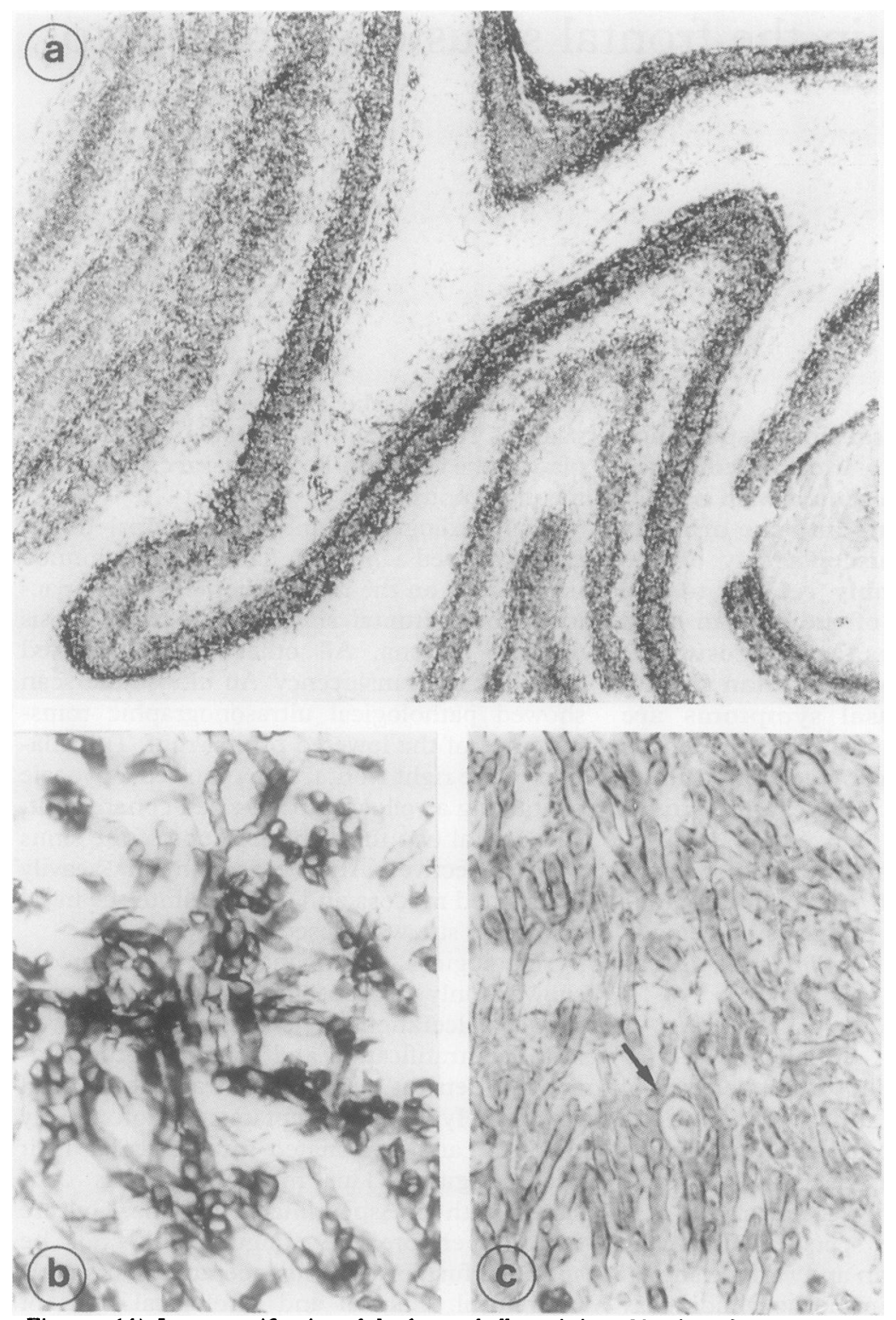

Figure (A) Low magnification of the fungus ball consisting of laminated mycelium (Grocott's methenamine silver); (B) Septated and dichotomous branching hyphae (Grocott's methenamine silver); (C) Conidial head detached from phialides (haematoxylin and eosin). brachycephalic shape of the human skull locating the frontal sinus far anterosuperiorly to the nasal cavity. By analogy, in dogs frontal sinus aspergillosis predominantly affects dolichocephalic breeds. Unlike its human homologue, it presents as destructive rhinosinusitis with clear nasal symptoms like purulent and bloody discharge. ${ }^{1}$

The eminent danger inherent to non-invasive aspergillosis is progression to invasiveness. Unlike pulmonary aspergillosis the rhinogenic form has a low tendency for haematogenous dissemination. It invades by continuity through resorptive bone defects or along perforating vessels. ${ }^{3}$ Orbital or intracranial invasion mainly arises from ethmoid or sphenoid sinuses, but may also start from the frontal sinus. Expansive growth or superinfection of the mycetoma eventually leads to epithelial destruction. The fungus itself cannot dissolve the keratin cytoskeleton of intact epithelia. Immunological impairment of the patient is a prerequisite only for fulminant aspergillosis. ${ }^{2}$

Isolated frontal sinus aspergillosis is symptomless until complications occur: in all reports orbital or intracranial encroachment produced the first clinical signs. ${ }^{5-9}$ The only case limited to one frontal sinus was discovered by roentgenography because of contralateral epistaxis. ${ }^{10}$ In this case only vague inconstant pressure sensations could be ascertained retrospectively. Trifling paranasal complaints and asymptomatic sinus opacity should therefore raise suspicion of paranasal aspergillosis. On the other hand, extensive brain abscesses may distract attention from adjacent structures: sinus opacity in such cases should actively be searched for. ${ }^{8}$

If paranasal aspergilloma is suspected endoscopy, tomography, and haemagglutination tests complete routine pretreatment diagnosis. Treatment is based on débridement and restoration of aeration. ${ }^{2610}$ Correct diagnosis of the surgical specimen requires histological and microbiological examination of intraluminal masses. ${ }^{2}$ Antimycotic drugs are useful in cases of frank invasion, recurrence, or defective immune response.

inantly affects the anterior ethmoid or the maxillary antrum. Posterior ethmoid, sphenoid, and particularly frontal sinus aspergillosis is rare, absent even in large series. ${ }^{2-8}$

This distribution is obviously determined by the topography of the access from the nasal cavity to the sinuses. Its main crossroad, the hiatus semilunaris, opening into the anterior ethmoid and the maxillary antrum, is located centrally in the middle nasal meatus. The openings into the posterior ethmoid, sphenoid, and frontal sinuses are located somewhat remotely from the main stream of respiration. The nasofrontal duct begins at the anterosuperior extremity of the ethmoidal infundibulum and is oriented in a ventro-cranial direction towards the frontal sinus. ${ }^{2}$ Analogous anatomical reasons predispose the maxillary antrum to aspergillosis: it is the lowest of all sinuses, with its ostium high in its medial wall, and may virtually act as collecting dish for secretion from ethmoid cells. The peculiarity of this paranasal topography arises from the
1 Mortellaro CM, Della Franca P, Caretta G. Aspergillus fumigatus, the causative agent of infection of the frontal 32:327-35.

2 Stammberger H, Jakse R, Beaufort F. Aspergillosis of the paranasal sinuses: $\mathrm{X}$-ray, diagnosis, histopathology and clinical aspects. Ann Otol Rhinol Laryngol 1984;93: 251-6.

3 Just E. Aspergillusabszeß des Großhirns. Mitteilüngen Grenzgebiete Medizin Chirurgie 1931;42:540-9.

4 Adams NF. Infection involving the ethmoid, maxillary and sphenoid sinuses and the orbit due to aspergillus fumigatus. Arch Surg 1933;26:999-1009.

5 Osborn DA. Mycotic infection of the frontal sinus. $f$ Laryngol 1963;77:29-33.

6 Warder F, Chikes PG, Hudson WR. Aspergillosis of the paranasal sinuses. Arch Otolaryngol 1975;101:683-5

7 Gupta KR, Udhayakumar B, Rao PB, Madhavan M, Das Gupta LR. Aspergilloma of the frontal bone. $\mathcal{F}$ Laryngol 1973;87:1007-11.

8 Bhalla D, Kumar S, Pal ND, Malhotra V, Dhingra PL Aspergilloma of the frontal lobe. Acta Neurochirurgica 1980;55:135-9.

9 Sekula J, Popielski L, Betkowski A. A case of aspergillosis involving the frontal sinus and orbit. Otolaryngologia Polska 1981;35:365-9.

10 Stevens MH. Aspergillosis of the frontal sinus. Arch Otolaryngol 1978;104:153-6. 